\title{
Body State Recognition for a Quadruped Mobile Robot
}

\author{
C. Kertész* and M. Turunen* \\ * Tampere Unit for Computer-Human Interaction (TAUCHI), University of Tampere, Tampere, Finland \\ *csaba.kertesz@ieee.org, markku.turunen@uta.fi
}

\begin{abstract}
The body states must be tracked by the onboard software on the robot to make good decisions. A human can pick up this machine or if the robot encounters anomalies (e.g. fall over) during locomotion, the state changes must be identified to execute the necessary responses. The authors of this paper developed a machine learning model which can recognize four states (normal, pick-up, fall over, poked) of a Sony AIBO robot. A deep neural network classifier with these predictors achieved $98 \%$ accuracy on unseen data and actual test runs on the robot proved the practical use with real-time execution speed. These properties made the proposed method a good candidate for adaption to other legged robots.
\end{abstract}

\section{INTRODUCTION}

It is essential to recognize the current robot state to execute the next actions for a mobile robot. To the best knowledge of the authors, the anomaly detection during locomotion is an underresearched topic in the robotics field and the majority of the literature was published in the previous decade, mainly related to the RoboCup competitions.

Hoffmann and Göhring [2] implemented collision detection for legged robots in Robocup. They compared the sensor readings with the actuator commands and their system could detect the collisions with varying success (50-90\% accuracy). A limitation was the dependency on the locomotion gait.

Spranger et al [6] developed a biologically inspired approach (Slow Feature Analysis) to recognize postures for a biped robot. Although their main objective was to recognize the basic static postures (e.g. lying, standing) and their state transitions, they could also detect when the robot was fallen. Their work did not include detailed analysis of the recognition accuracy.

Meriçli et al [7] proposed a collision detection framework for omnidirectional motion of a quadruped robot. They examined the temporal accelerometer readings in the frequency domain for regularities (normal motion) and novel situations (collisions). The robot built a probabilistic model while walking without obstruction and used this model to determine any unfamiliar pattern. Their experiments shown quick and successful detection of collisions.

Goswami et al [8] worked on the fall detection for humanoid robots. Their goal was to change the default fall direction of the robot during the fall event to avoid delicate objects or a person. They proposed the fall trigger boundary (FTB) for fall prediction in the robotic systems which is a boundary in the feature space of the robot variables (sensor values, angular momentum). The robot can maintain its balance inside the boundaries though it will definitely fall once the boundary is passed. There was no implementation example for this approach in the simulation environment of [8], but this boundary is learned with machine learning modeling for Sony AIBO in this paper.

Tam and Kottege [9] introduced a robust fall prevention system for bipedal robots. Their Robotis OP2 robot used a walking stick for recovery actions to keep its balance by extending the support polygon on the ground. The Fall Classifier in Robotis OP2 was based on the famous inverted pendelum model to keep the centre of mass inside the support polygon determined by the feet on the ground. The system tracked the inertial measurement unit readings, the angular velocities and the fall angle relative to the walking stick and a fall event happened when the robot control detected unrecoverable instability of the walking sequence. Contrary to [9], the fall detection in this paper was treated like a pattern classification problem in a different approach.

As it can be seen, the literature concentrated on the collision detection, but none of them modeled two situations during human-robot interactions. First, when a human threatens a robot by poking it, and second, when the robot is picked up by person and transported to a new location. The authors of this paper built a machine learning (ML) model to detect these situations and the fall over events for a Sony ERS-7 robot (Fig. 1). The following sections describe how the dataset was created, an optimal ML model was selected and finally tested on the robot.

\section{DATASET}

A dataset was collected to detect robot state changes. When the robot operates without anomalies and human intervention, it is in normal state. The fall over events must be detected to recover in these situations and the robot may be under threat if a human starts to poke from side. The robot must also recognize if it was picked up by a person and it is being transported to a new location. These four states (normal, fall over, poked, picked up) must be recognized by a machine learning method in this paper, therefore, a dataset was collected with a Sony ERS7 robot. After the feature vectors were generated (see in the next section), 76535 samples were in the dataset: 52612 samples for normal, 12831 for picked up, 1323 for fall over and 9769 for poked state. The normal state has by far the most samples since it involves all activities of a legged robot: walking, sitting, lying, standing, object manipulation, human-robot interaction etc. The picked up 
and poked sample counts are moderate because they can be easily collected. However, the fall over can stress the robot body regardless of the safe landing on a soft ground thus it cannot be repeated many times for data collection. Furthermore, one fall over generates few samples per event caused by the short duration and these circumstances result a skewed label with 1323 samples.

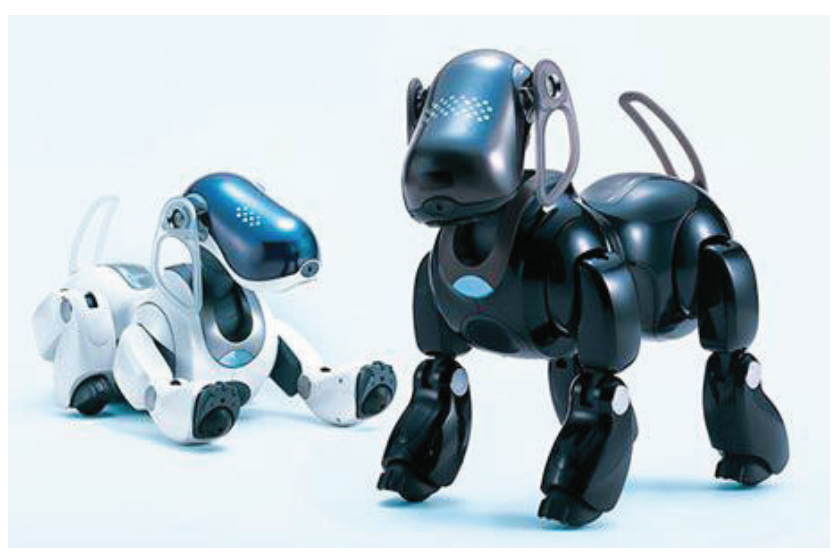

Fig. 1: Sony ERS-7 robot dogs

The samples were separated randomly to training $\left(S_{T}\right)$ and validation sets $\left(S_{V}\right)$ because the former can be used to run 10-fold cross-validation and train a model while the former is useful to evaluate the built model with unseen data. $57.52 \%$ of the samples were part of the training set: 31607 for normal, 5855 for picked up, 694 for fall over and 5870 for poked states. $42.47 \%$ of the samples went in $S_{V:}: 21005$ normal samples, 6976 picked up samples, 629 fall over samples and 3899 poked samples.

\section{FeAture Vector}

The tactile sensors have been widely used for multiple purposes for mobile robots to recognize the directional bias during locomotion [1], collision detection [2] or surface and slope detection [3]. The authors of this paper invented some predictors for body state estimation with a Sony AIBO robot which has a low-cost accelerometer in the torso with a $120 \mathrm{~Hz}$ sampling rate. A feature vector representation of the samples is needed for traditional machine learning methods, therefore, these vectors were generated by a $270 \mathrm{msec}-$ long sliding window with 32 accelerometer values per axis and locomotion states. Let us denote the feature vector and the sliding window:

$$
\begin{aligned}
& F V=\left\{f v_{0}, f v_{1}, \ldots, f v_{12}\right\}, \\
& A_{w}=\left\{a_{t}, a_{t+1}, \ldots, a_{t+31}\right\},
\end{aligned}
$$

where $F V$ composed of 13 numbers and $A_{w}$ contains sensor values for one axis ( $\mathrm{x}, \mathrm{y}$ or $\mathrm{z})$. Some statistics were computed for each accelerometer axis separately, but the formulas were otherwise identical. These predictors were defined as:

$$
f v_{0}=i q r\left(A_{w, x}\right), f v_{1}=i q r\left(A_{w, y}\right), f v_{2}=i q r\left(A_{w, z}\right) \text {, }
$$

$$
\begin{aligned}
& f v_{3}=\min \left(A_{w, x}\right), f v_{4}=\min \left(A_{w, y}\right), f v_{5}=\min \left(A_{w, z}\right), \\
& f v_{6}=\max \left(A_{w, x}\right), f v_{7}=\max \left(A_{w, y}\right), f v_{8}=\max \left(A_{w, z}\right),
\end{aligned}
$$

where $f v_{0-2}$ are the interquartile ranges of each axis, $f v_{3-5}$ are the minimum and $f v_{5-7}$ are the maximum values. These predictors describe how much the body oscillated inside the sliding window. The rest four predictors contained information about the locomotion state of the robot:

$$
\begin{aligned}
& f_{9}=\left\{\begin{array}{ll}
1: & W_{\text {forward }, t}>0 \mathrm{msec} \\
0 & : \text { otherwise }
\end{array},\right. \\
& f v_{10}=\left\{\begin{array}{ll}
1: & W_{\text {backward }, t}>0 \text { msec } \\
0: & \text { otherwise }
\end{array}\right. \text {, } \\
& f v_{11}=\left\{\begin{array}{ll}
1: & W_{\text {turnleft, }}>0 \mathrm{msec} \\
0 & : \text { otherwise }
\end{array},\right. \\
& f v_{12}=\left\{\begin{array}{ll}
1: & W_{\text {turnright, },}>0 \mathrm{msec} \\
0 & : \text { otherwise }
\end{array},\right.
\end{aligned}
$$

where $f v_{9}$ is non-zero if the robot walks forward, $f v_{10}$ is non-zero if the robot walks backward and $f_{11-12}$ are related to the turning directions respectively. $W_{\text {forward, }, \text {, }}$ $W_{\text {backward, },}, W_{\text {turnleft, } t}$ and $W_{\text {turrnright, } t}$ are the elapsed time in a certain action and they are beneficial when the locomotion state and the accelerometer statistics are contradicting each other. For example, the robot walks forward and a human picks it up. While $W_{\text {forward, } t}$ indicate forward walk in the feature vector, $f v_{0-8}$ statistics will be out of the ranges of ordinary forward walk.

FV was defined by statistics of accelerometer values and locomotion actions. The following section will describe the classifiers which were trained by FV.

\section{Classifiers}

There is no best classifier for all problems and different methods can achieve satisfactory results. After the samples of the training set $\left(S_{T}\right)$ were standardized, six classifiers were evaluated by 10 -fold cross-validation (CV) whose results are shown on Fig. 2. The naïve Bayes (NB) and support vector machine (SVM) had the worst results with $73.79 \%$ and $76.46 \%$. The SVM classifier used linear kernel with dual coordinate descent method [4] and its hyperparameter was $\mathrm{C}=0.1$. The k-nearest neighbor $(\mathrm{KNN})$ had the best accuracy with $99.60 \%$, followed by the decision tree (DT) classifier with $99.44 \%$. DT had two hyperparameters, the max depth was set to 20 and the tree node sample limit was 1 . The random forest (RF) had lower result (98.09\%) than DT which can be caused by the task complexity. A random forest is a collection of decision trees and RF can learn a problem better than one DT if the learning capacity in one DT is not enough for the complexity.

The deep learning is a major research area in machine learning nowadays and breakthrough results appear with this approach in many robotics fields. The deep neural network (DNN) in this study has two hidden layers with 20-20 neurons and leaky ReLu activation functions. The output layer has 4 neurons with softmax function to calculate the classification result. The adagrad adaptive 
gradient algorithm was run on the network with batch size 64 for 50 epochs. Although somebody can argue that this network topology is not bigger than the classical neural networks from the nineties, but the recent developments in the learning algorithms and other parts made the new networks outperform the old in even simple tasks. The authors of this paper found small neural networks surpassing traditional methods with handcrafted features lately in [5]. DNN had a moderate result $(88.95 \%)$ in the 10 -fold cross-validation.

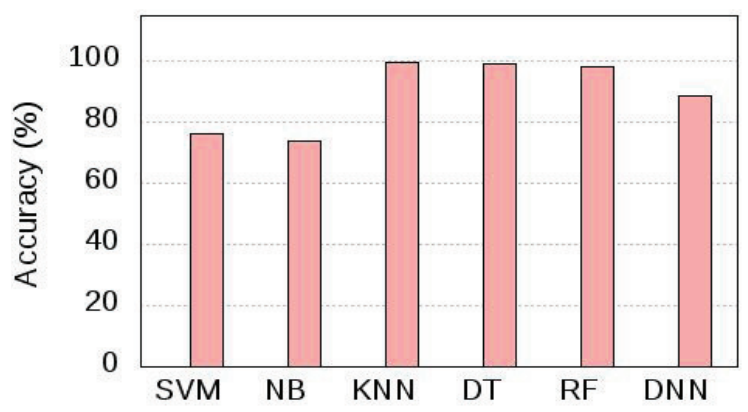

Fig. 2: 10-fold cross-validation results of six classifiers: support vector machine (SVM), naïve Bayes (NB), knearest neighbor $(\mathrm{KNN})$, decision tree (DT), random forest (RF) and deep neural network (DNN).

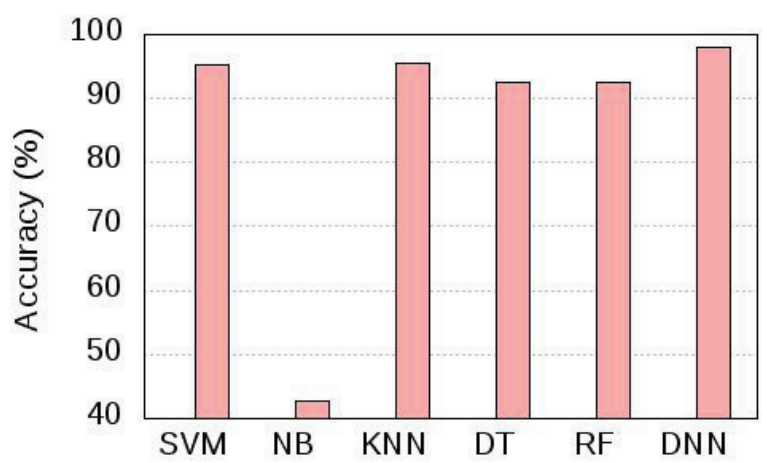

Fig. 3: Model evaluation results with six classifiers: support vector machine (SVM), naïve Bayes (NB), knearest neighbor $(\mathrm{KNN})$, decision tree (DT), random forest (RF) and deep neural network (DNN).

Table I. Confusion matrix of deep neural network model results. The rows show the real body states and the columns how they were classified.

\begin{tabular}{|l|l|l|l|l|}
\hline & Normal & Picked up & Fall over & Poked \\
\hline Normal & 99.28 & 0.18 & & 0.53 \\
\hline Picked up & 1.90 & 97.40 & & 0.68 \\
\hline Fall over & 13.35 & & 86.64 & \\
\hline Poked & 3.61 & 2.30 & & 94.07 \\
\hline
\end{tabular}

Table II. Memory and runtime requirements of five models.

\begin{tabular}{|l|l|l|l|l|l|}
\hline & SVM & KNN & DT & RF & DNN \\
\hline $\begin{array}{l}\text { Model } \\
\text { accuracy }\end{array}$ & $95.35 \%$ & $95.42 \%$ & $92.59 \%$ & $92.59 \%$ & $98.01 \%$ \\
\hline $\begin{array}{l}\text { Memory } \\
\text { consumption }\end{array}$ & $2 \mathrm{~KB}$ & $2.5 \mathrm{MB}$ & $117 \mathrm{~KB}$ & $181 \mathrm{~KB}$ & $28 \mathrm{~KB}$ \\
\hline $\begin{array}{l}\text { Execution } \\
\text { time }\end{array}$ & $2 \mathrm{usec}$ & $1.6 \mathrm{msec}$ & $3 \mathrm{usec}$ & $3 \mathrm{usec}$ & $20 \mathrm{usec}$ \\
\hline
\end{tabular}

After the cross-validation was run on the training set $\left(S_{T}\right)$, models were built with the whole $S_{T}$ set and their performance were evaluated with unseen data $\left(S_{V}\right)$ in the next section.

\section{Model SELECTION}

Models were built upon the training set $\left(S_{T}\right)$ and the accuracies were calculated by the samples of the validation set $\left(S_{V}\right)$. Fig. 3 shows the results which did not follow the trends of the cross-validation in Fig. 2. This phenomenon is originated in the fact that the crossvalidation gives an estimation about the real classifier performance on unseen data and the reality can be far from the CV accuracies. As it can be seen, SVM had the second worst $\mathrm{CV}$ result, but it achieved third best accuracy with $95.35 \%$. NB had the worst result in Fig 2. and this classifier collapsed in the model evaluation with $42.65 \%$ accuracy. The k-nearest neighbor had the best CV value, but it reached the second place in Fig. 3 with $95.42 \%$. DT and RF had good cross-validation results and they achieved performance over $90 \%$ with $92.59 \%$ in Fig. 3 . The deep neural network was not the best in the crossvalidation round, nevertheless, it was the top-performer in the model evaluation with $98.01 \%$ accuracy. This result suggests, similar to [5], that the new generation of neural networks can outperform the old methods by margin in even small problems. While the traditional algorithms have still the advantage of the quicker learning speed and a possible smaller memory footprint, it is a disadvantage that they need hyperparameter optimization even for small problems to achieve the best performance. On the other hand, the authors experienced that deep neural networks can be trained with handcrafted features, default hyperparameters and simple multilayer perceptron topology to learn a training set with good generalization power out of the box.

The confusion matrix of the DNN model is shown in Table I. As expected, the normal samples had the best accuracy $(99.28 \%)$ since this state had the most samples in the dataset. The picked up and poked labels were recognized well with $97.40 \%$ and $94.07 \%$ accuracies although 1-3\% misclassifications happened in a few cases. The fall over had the least, $86.64 \%$ accuracy and $13.35 \%$ of these samples were misclassified to normal state. Since none of the other labels were misclassified as fall over and fall over samples were misclassified only as normal state (yellow and gray cells in Table I), the authors believe that the predictors were strong to identify the fall over situation. But when a fall over event starts, the samples are similar to the normal status before the robot turns over its center of gravity and falls with accelerating speed. Since 
the sliding window is $270 \mathrm{msec}$ long to compute a feature vector, some time is necessary until the fall over event dominate the accelerometer statistics inside the sliding windows. This is a possible explanation for this misclassification.

The memory requirements and the execution times of all classifiers are listed in Table II except naïve Bayes which failed to build a satisfactory model. The k-nearest neighbor has the highest memory consumption and prediction time because this method stores all training samples in the memory and use them in each prediction step. The decision tree and random forest had moderate memory usage, their execution times were quick, but their accuracies were relative low compared to the other classifiers. The SVM achieved the best memory and execution time results, therefore, this classifier is recommended for robots with microcontrollers where every $\mathrm{KB}$ and microsecond count. However, the deep neural network had small resource needs paired with the best model accuracy thus this classifier is the best choice to run in microsecond-scale on any embedded CPU.

\section{Evaluation on The Robot}

The deep neural network outperformed the other classifiers with unseen data from the validation set $\left(S_{V}\right)$. However, this evaluation does not depict exactly how the model will work under real conditions. To address this problem, the DNN model was deployed to a Sony ERS-7 to inspect how the classifier behaves in some typical situations without additional filtering.

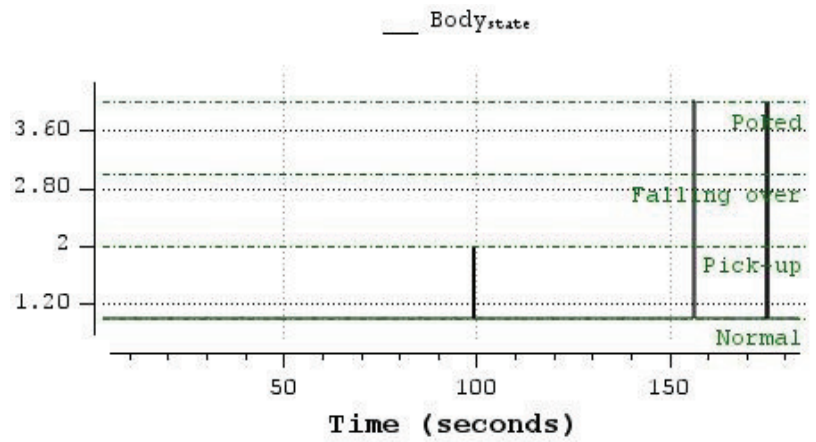

Fig. 4: A normal runtime with basic postures and locomotion. External events did not cause any disturbances for the robot.

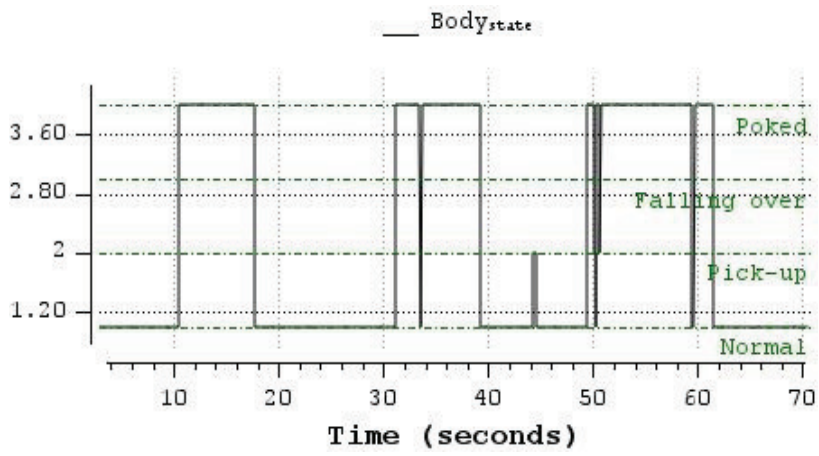

Fig. 5: The robot was poked in sitting, standing and lying postures.

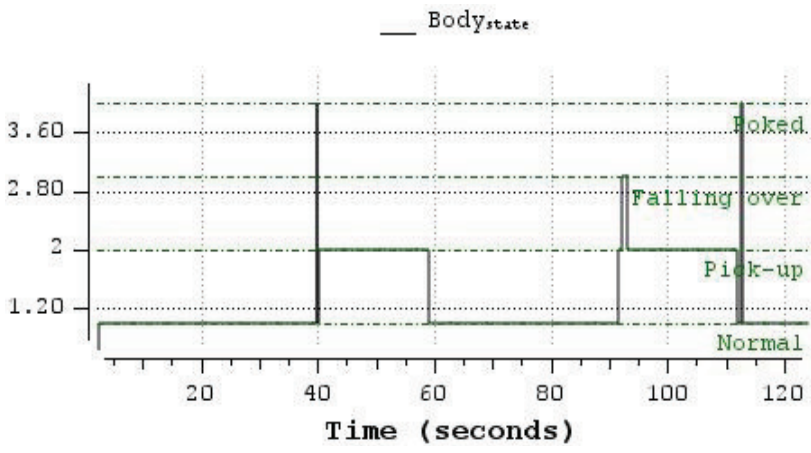

Fig. 6: The robot was picked up from lying posture and

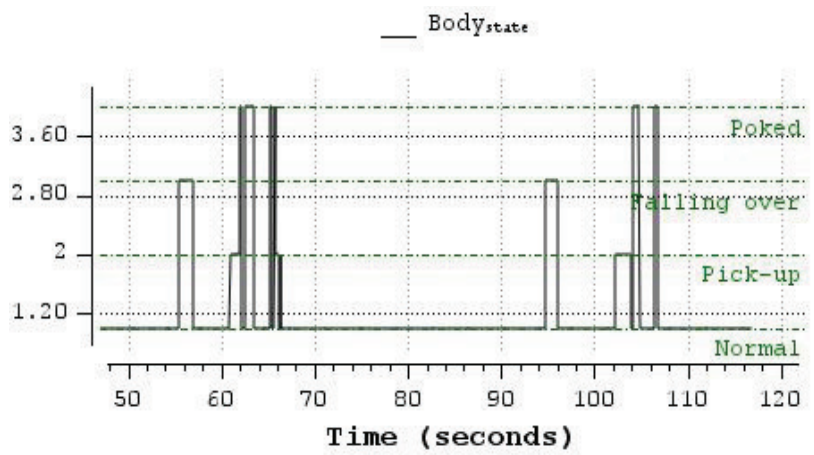

Fig. 7: An example runtime when the robot falls.

In the first case, the robot completed usual activities without any disturbances. After transitioning between lying, sitting and standing postures, the robot walked forward, backward and turned in both directions. The recognized body states are shown on Fig. 4. As it can be seen, the classifier detected the normal operation correctly almost all the time. There were three very short periods when picked up and poked events were identified for a moment.

The robot can be disturbed by poking in static postures (sitting, lying, standing). Fig. 5 shows that the robot was poked between 10-18 seconds in lying posture. After the transition was finished to sitting posture, the robot was poked between 31-39 seconds. And after standing up, the robot was tossed again between 50-62 seconds. As we can see, the long poking events were recognized correctly and only a very short picked up event was misclassified around 45 seconds during the transition between sitting and standing postures.

Fig. 6 demonstrates picked up events. The robot was in lying posture when a person picked it up and walked around between 40-60 seconds. The robot was placed back to the floor later before it stood up and started to walk forward. The person picked it up again and carried between 91-113 seconds. The picked up states were recognized correctly except two short poked and one fall over events.

Fig. 7 shows two fall over events during locomotion. The robot fallen to the left side while it walked forward after 55 seconds and to the right side after 95 seconds. Till the robot was turned back to lying posture, the misclassified poked and picked events happened for longer periods compared to the previous situations. These events can be easily suppressed until the lying posture is reached after falling down. 
As Fig. 4-7 showed the deep neural network model was robust and misclassifications did not happen for significant periods. These results suggest that the model can recognize all states correctly under realistic conditions after a minimal filtering.

\section{CONCLUSION}

This paper described a machine learning approach to detect sudden changes in the robot state such as fall over, poking and being picked up by a human. A legged robot was used for data collection and several classifiers were challenged to find the best performing for embedded use. The deep neural network achieved 98\% accuracy and its low resource usage made suitable for running in embedded robotics systems. Although the built model processed each data frame separately without temporal fusion, actual tests on the robot shown that the proposed method was applicable under real circumstances with minimal false positives.

Future work can include the further analysis of temporal machine learning approaches (e.g. recurrent neural networks) with raw sensor data since the current solution operates with handcrafted features. Another direction can be to add new states for the model or study the applicability of the predictors with other mobile robots.

\section{REFERENCES}

[1] D. J. Huang and W. C. Teng, "A Gait Based Approach to Detect Directional Bias of Four-Legged Robots' Direct Walking Utilizing Acceleration Sensors," Intl. Conf. on Knowledge-Based and Intelligent Information and Engineering Systems, 2007, pp. 681688.

[2] J. Hoffmann and D. Göhring, "Sensor-actuator-comparison as a basis for collision detection for a quadruped robot," in Robot Soccer World Cup, 2005, pp. 150-159.
[3] Y. Chen, C. Liu, and Q. Chen, "A vestibular system model for robots and its application in environment perception," Intl. Conf on Computing, Control and Industrial Engineering (CCIE), 2010, pp. 230-235.

[4] C. J. Hsieh, K. W. Chang, C. J. Lin, S. S. Keerthi, and S. Sundararajan, "A dual coordinate descent method for large-scale linear SVM," 25th Intl. Conf. on Machine Learning, 2008, pp. 408-415.

[5] C. Kertesz and M. Turunen, "Common Sounds in Bedrooms (CSIBE) Corpora for Sound Event Recognition of Domestic Robots,", in Intelligent Service Robotics, forthcoming/in press, 2018.

[6] M. Spranger, S. Höfer, and M. Hild, "Biologically inspired posture recognition and posture change detection for humanoid robots," IEEE Intl. Conf on Robotics and Biomimetics (ROBIO), 2010, pp. 562-567.

[7] T. Meriçli, C. Meriçli, and H. L. Akin, "A robust statistical collision detection framework for quadruped robots," in Robot Soccer World Cup, 2008, pp. 145-156.

[8] A. Goswami, S. K. Yun, U. Nagarajan, S. H. Lee, K. Yin, S. Kalyanakrishnan, "Direction-changing fall control of humanoid robots: theory and experiments," in Autonomous Robots, 36(3), 2014, pp. 199-223.

[9] B. Tam, N. Kottege, "Fall avoidance and recovery for bipedal robots using walking sticks," in Australasian Conf. Robotics and Automation, 2016. 